\title{
ERRATUM
}

\section{Mispronunciation of Japanese singleton and geminate stops by Korean and Taiwanese Mandarin speakers, [Acoustical Science and Technology 42(2), 73-82 (2021)]}

\author{
Kimiko Yamakawa ${ }^{1, *}$, Shigeaki Amano ${ }^{2, \dagger}$ and Mariko Kondo ${ }^{3, \ddagger}$ \\ ${ }^{1}$ Faculty of Contemporary Culture, Shokei University, \\ 2-8-1 Musahigaoka, Kikuyo, Kikuchi-gun, Kumamoto, 861-8538 Japan \\ ${ }^{2}$ Faculty of Human Informatics, Aichi Shukutoku University, \\ 2-9 Katahira, Nagakute, 480-1197 Japan \\ ${ }^{3}$ School of International Liberal Studies, Waseda University, \\ 1-6-1 Nishiwaseda, Shinjuku-ku, Tokyo, 169-8050 Japan
}

The following corrections should be made to the article entitled "Mispronunciation of Japanese singleton and geminate stops by Korean and Taiwanese Mandarin speakers," by Yamakawa, K., Amano, S. and Kondo, M., which appeared on pp. 73-82 in 42(2) 2021 issue of Acoustical Science and Technology. Equation (1) on page 76 is incorrect. The correct version is $0.0889 x-y+61.4=0$.

*e-mail: jin@ @ shokei-gakuen.ac.jp

†e-mail: psy@asu.aasa.ac.jp

‡e-mail: mkondo@waseda.jp

[doi:10.1250/ast.43.81] 